\title{
MARTIN KINTZINGER
}

\section{ENTRE EXERCICE DU POUVOIR ET DROIT DES GENS}

\author{
La diplomatie de l'empereur Sigismond envers la France
}

\section{Paris et Berlin}

"Paris et Berlin proposent d'harmoniser la fiscalité des entreprises«, c'est ainsi que l'on a récemment annoncé (fin mai 2004) une information de presse concernant un autre chapitre de la coopération franco-allemande dans le cadre de la Communauté européenne ${ }^{1}$. Dans ce cas, il s'agit des finances; mais dans de nombreux autres domaines de la politique gouvernementale, les ministres et les diplomates des deux côtés, souvent même les chefs des gouvernements eux-mêmes sont en contact étroit. Les fondements juridiques pour de telles coopérations sont réglés clairement à l'intérieur de l'Europe et entre les deux États. Ils reposent sur le fait que les pays partenaires sont au même niveau non seulement dans un équilibre momentané des forces, connu comme programme politique depuis le début des Temps modernes ${ }^{2}$, mais surtout fondamentalement et durablement.

Tous les pays de la Communauté européenne sont de cette manière mis au même rang, par des contrats qui existent et que l'on retrouvera dans une constitution commune. Le grand public s'étonne toujours que quelques pays membres de la Communauté empêchent l'ordre constitutionnel projeté ${ }^{3}$. Ils expliquent leur refus en disant que la répartition des forces politiques est déséquilibrée - et surtout que la France et l'Allemagne exercent une primauté de fait qui serait confirmée par la constitution. L'harmonie entre Paris et Ber-

1 www.lemonde.fr du 29 mai 2004: Arnaud LEPARMENTIER, Paris et Berlin proposent d'harmoniser la fiscalité des entreprises. Je remercie mon collègue Philippe Genequand (université de Genève) pour une lecture critique de mon texte.

2 Voir Arno STROHMEYER, Theorie der Interaktion. Das europäische Gleichgewicht der Kräfte in der frühen Neuzeit, Vienne, Cologne, Weimar 1994; Heinz DUCHHARDT, Balance of power and pentarchie, 1700-1785, Paderborn et al. 1997 (Handbuch der Geschichte der internationalen Beziehungen, 4).

${ }^{3}$ www.spiegel.de: dossier $»$ Der zähe Kampf um das Jahrhundertwerk [i.e. la constitution de l'Union européenne; articles dès automne 2003]«. Cf. le jugement du premier ministre britannique, Tony Blair, du 19 juin 2004, sur "l'arrogance du couple franco-allemand“. 
lin est-elle en vérité une hégémonie, la politique en Europe suit-elle le droit ou le pouvoir?

Cet exposé est subdivisé en deux parties. On esquissera d'abord le développement de la manière de penser selon le droit des gens au Moyen Âge jusqu'au recommencement, autour de 1400; on retracera ensuite la politique de l'empereur Sigismond en Europe de l'Ouest comme exemple d'une politique internationale moderne.

\section{Exercice du pouvoir et droit des gens}

\section{Droit et violence}

Ce n'est pas un hasard si les événements dramatiques actuels - la guerre des Balkans et en Irak - ont été intensivement commentés par la presse selon le slogan "comme au Moyen Âge «'. La guerre des Balkans est le sujet d'un livre auquel le publiciste français Alain Mine donna le tire "Le nouveau Moyen Âge«. Jean-Marie Moeglin a formulé en 2001 une réponse pertinente en écrivant que le reproche d'excès barbare de pouvoir et de violence devait être nuancé .

Longtemps avant le siècle des lumières - qui n'a pas pu protéger les Temps modernes de la violence barbare - le Moyen Âge connaissait déjà souvent des débuts même "modernes" d'une communication internationale dans la diplomatie et la politique. Une telle communication se déroulait dans beaucoup de cas semblablement à aujourd'hui, par des entretiens et des contacts personnels, par des rencontres entre souverains et par des négociations formelles et amples. Les succès et les échecs étaient alors aussi proches qu'aujourd'hui'.

\section{Internationalité et droit des gens}

Il s'y ajoutait un répertoire de procédures différencié d'actions cérémonieuses et rituelles qui, aujourd'hui, ne sont plus en vigueur que d'une manière rudimentaire (et inconnues à beaucoup). Mais ces procédures dans la vie diploma-

\footnotetext{
4 Wolfgang REINHARD, Geschichte der Staatsgewalt. Eine vergleichende Verfassungsgeschichte Europas von den Anfängen bis zur Gegenwart, Munich 2002, p. 305 sq.

${ }^{5}$ Alain MINC, Le nouveau moyen âge, Paris 1993.

${ }^{6}$ Jean-Marie MOEGLIN, Von der richtigen Art zu kapitulieren: Die sechs Bürger von Calais (1347), dans: Hans-Henning KoRTÜM (dir.), Krieg im Mittelalter, Berlin 2001, p. 141-166, ici p. 160.

${ }^{7}$ REINHARD, Staatsgewalt (voir n. 4), p. 370-372.
} 
tique du Moyen Âge avaient une importance prédominante. Afin de pouvoir agir efficacement, il était nécessaire d'avoir une mise en scène de la représentativité dans le cadre de l'hégémonie pour harmonie et hégémonie, pouvoir et droit, regnum et imperium.

La recherche moderne d'actions rationnelles et désirées doit avoir lieu dans le cadre de la culture des signes pour pouvoir comprendre la réalité historique - à l'intérieur des regna comme à l'extérieur, parce que les cours de tous les regna de l'Europe chrétienne étaient liées à la même culture et se servaient des mêmes formes de la communication symbolique ${ }^{8}$.

À ce propos non seulement le jeu de l'intention politique et la représentation cérémonielle - comme expression du pouvoir, mais aussi le fondement juridique donné et choisi des actions mutuelles - était important. Il ne faut pas se méprendre, la culture de la cour n'est pas à comprendre comme un »droit fixé« de la même façon que l'est l'ordre juridique, et selon lequel on pourrait agir en politique. Il fut traité de manière similaire selon les formes de procédures de la culture de cour: sur la base de la connaissance acquise et de l'expérience obtenue.

On ne peut pas nier qu'un droit des gens donné, défini selon le droit positif et réalisé par des contrats n'existe que depuis le début des Temps modernes". La chose, les valeurs et les formes du droit coutumier de la communication internationale étaient, quand-même, présentes au Moyen Âge - à côté des normes écrites du droit romain, elles furent appliquées en pratique et réfléchies d'une manière théorique. Il n'est pas important pour notre question de savoir si la pratique et la théorie (à la différence des normes du droit positif) ne sont pas expressément nommées comme ius gentium ${ }^{10}$.

\section{Imperium et regnum}

La requête centrale de la littérature des traités est la relation entre regnum et imperium et la question d'une supériorité possible, prétendue ou contestée d'un pouvoir princier (de l'empereur) sur un autre (royal). Il s'agissait toujours

${ }^{8}$ Cf. l'article de Gerald Schwedler dans ce volume et aussi: Martin KINTZINGER, Der weiße Reiter. Formen internationaler Politik im Spätmittelalter, dans: Frühmittelalterliche Studien 37 (2003), p. 315-353; ID., Politische Westbeziehungen des Reiches im Spätmittelalter: Westliche Kultur und Westpolitik unter den Luxemburgern, dans: Joachim EHLERS (dir.), Deutschland und der Westen Europas im Mittelalter, Ostfildern 2002 (Vorträge und Forschungen, 61), p. 423-455.

${ }^{9}$ Je remercie ma collègue Barbara Stollberg-Rilinger (université de Münster) pour la collaboration et des informations sur le développement du droit des gens dans le XVII ${ }^{e}$ siècle.

${ }^{10}$ Wilhelm G. GREWE (dir.), Fontes historiae iuris gentium. Quellen zur Geschichte des Völkerrechts. Sources relating to the history of the law of nations, t. 1: $1380 \mathrm{v}$. Chr.-1493, Berlin, New York 1995. Au droit romain: Institutionen Justinians [de 533], Stuttgart 1903, $n^{\circ} \mathrm{C}$ III lb, p. 168-172. 
d'une comparaison des rangs entre imperium et regnum ou regna, entre imperator et rex et ici en particulier entre l'empereur de l'Empire romain germanique et le roi de France. Le légiste bourguignon Jean de Blanot a formulé à la fin du XIII ${ }^{e}$ siècle la théorie: Rex Franciae in regno suo princeps est, dans la pratique politico-diplomatique en abrégé: Rex Franciae Imperator in regno suo. Ainsi chaque supériorité d'un autre potentat, même celle de l'empereur envers le roi de France, est refusée d'une manière catégorique ${ }^{11}$. L'interprétation impériale de la phrase se retrouvait en même temps chez le célèbre légiste et particulièrement efficace du Moyen Âge, celui de Baldus de Ubaldis venu de Florence, de Padoue et de Pavie et décédé en 1400: Rex in regno suo est imperator regni sui ${ }^{12}$.

Dans le contexte de notre sujet, une toute autre ligne de tradition nous mène à Baldus. Pour le développement de la pensée du droit des gens à la fin du Moyen Âge, il existait deux fondements essentiels: d'abord les pensées développées par Augustin, et précisées par Thomas d'Aquin, selon lesquelles le droit divin (ius divinum) est suivi d'un droit naturel de tous les hommes (lex naturalis, ius naturale) et ensuite qu'une protection doit être garantie par le droit canonique pour assurer l'inviolabilité matérielle et corporelle des attachés de légation (legati inviolabiles) ${ }^{13}$. La première affirmation reposait sur la théorie du droit des gens, la deuxième sur la pratique diplomatique. Après que le terme et la compréhension du ius gentium - encore existants chez Isidore s'étaient perdus, on les a redécouverts lors des combats des partis au XIV ${ }^{\mathrm{e}}$ siècle, surtout entre le pouvoir temporel et le pouvoir spirituel des papes ${ }^{14}$.

Ce fut Baldus qui (également au XIV ${ }^{\mathrm{e}}$ siècle) à partir d'une raison donnée naturelle (naturalis ratio) conclut que les peuples (populi) et le gouvernement d'un peuple (regimen populi) n'avaient pas besoin d'une autorité supérieure lors de leur législation voulue. Les peuples sont directement liés au droit des gens et ceci vaut également pour le gouvernement d'un peuple (populi sunt de iure gentium ergo regimen populi est de iure gentium).

${ }^{11}$ Henri GiLles, art. Blanot, Jean de, dans: Lexikon des Mittelalters, vol. 2, Munich 1983, col. 264sq.

${ }_{12}$ Peter Weimar, art. Baldus de Ubaldis, dans: Lexikon des Mittelalters, vol. 1, Munich 1980, col. 1375sq.; Dieter MERTENS, Geschichte der politischen Ideen im Mittelalter, dans: Hans FENSKE et al. (dir.), Geschichte der politischen Ideen. Von Homer bis zur Gegenwart, Francfort/M. 1991 (Königstein 1981), p. 143-238, ici p. 206.

${ }^{13}$ GrEwE (dir.), Fontes (voir n. 10), Augustin: $n^{\circ}$ D I 6a, p. 270sq., Thomas: $n^{\circ}$ D I 6c, p. 272sq. La protection des députés au droit canonique: n D XII 2d, p. 664 (päpstliches Mahnschreiben, 1272). Sur le même sujet est à paraître une thèse de Claudia Zey (université de Zurich). Sur la connexion du droit naturel et droit des gens chez Thomas d'Aquin: REINHARD, Staatsgewalt (voir n. 4), p. 103sq., 382sq.

${ }^{14}$ Cf. Karsten PLÖGER, Die Entführung des Fieschi zu Avignon (1340). Zur Entwicklung der diplomatischen Immunität in der Frühphase des Hundertjährigen Krieges, dans: Francia 30/1 (2003), p. 73-105, ici p. 102sq. 


\section{Imperator et mediator}

Si l'on prend l'imperator dans le cadre étroit des regna, cela signifie donc par là-même qu'on lui confère des tâches qui vont au-delà des frontières internationales. Quand à la fin du Moyen Âge on se mit à redéfinir la relation de l'imperium et des regna en prenant en considération le ius gentium, la prétention de validité universelle de l'empereur était entre temps oubliée. De fait, la dignité impériale de l'Empire romain germanique était inséparablement liée à la couronne de l'Empire allemand, seul le roi allemand élu pouvait être couronné empereur et il obtenait d'office ainsi plus de dignité et d'autorité que les autres reges, mais seulement plus d'autorité et plus de validité, mais de fait pas plus de pouvoir.

Plus qu'auparavant, il existait autour de 1400 la demande d'une telle autorité $^{15}$. La menace permanente des Turcs, les multiples disputes entre princes chrétiens et les besoins de conciles généraux signifiaient une charge incomparable. On avait besoin dans cette situation d'une instance internationale de médiation et d'arbitrage. Peu de choses depuis des siècles avaient changé dans l'activité et la fonction des médiateurs (mediatores), ceci vaut également pour les succès et les échecs de leurs actions. "D'une manière classique - quand il s'agissait de conflits internationaux - le pape était un mediator approprié, qui était considéré comme neutre et qui disposait d'une autorité universelle. Il n'en était plus question au temps du schisme. La concurrence des siècles durant entre le pape et l'empereur était abolie dans cette situation. Sans la médiation universelle couronnée de succès de l'empereur, on ne pouvait pas s'attendre une réussite dans la négociation conciliaire de la causa unionis, de l'unification de l'Église et du choix d'un seul pape reconnu.

Malgré cela, la littérature des traités du XIV et du XV siècle a pris un autre chemin et s'est poursuivie avec les réflexions de Baldus concernant le droit des gens dans une direction capricieuse et absolument moderne. Pierre Dubois déjà au début du XIV ${ }^{\mathfrak{e}}$ siècle a réfléchi à l'instauration d'une cour d'arbitrage, devant laquelle pouvaient porter plainte et défendre ceux auxquels on avait auparavant accordé d'être des princes sans reconnaissance d'une autorité supérieure de règne (principes superiores in terris non recognoscentes) ${ }^{16}$. C'est le roi de Bohême, Georg Podiebrad, directement menacé par ses adversaires, qui est allé le plus loin avec son plan de la paix de $1462^{17}$. Bien qu'il n'ait pas cité le droit des gens, il a esquissé un tribunal valide de droit international. Le plan

${ }^{15}$ Cf. Ferdinand SEIBT, Winfried EbERhaRD (dir.), Europa 1400. Die Krise des Spätmittelalters, Stuttgart 1984.

${ }^{16}$ GREWE (dir.), Fontes (voir n. 10), $n^{\circ}$ D IX 3a, p. 555sq.: Pierre Dubois (plan de la paix, 1306); Otto Gerhard OEXLE, art. Dubois, Pierre, dans: Lexikon des Mittelalters, vol. 3, Munich 1986, col. 1433sq.

${ }^{17}$ GrewE (dir.), Fontes (voir n. 10), $\pi^{\circ}$ D I 3b, p. 254-264; Josef MaCEK, art. Georg von Podiebrad, dans: Lexikon des Mittelalters, vol. 4, Munich 1989, col. 1275sq. 
de Podiebrad que l'on peut sans hésiter considérer comme un témoin d'une politique européenne des Temps modernes, a connu très banalement un échec dans la pratique, le refus des princes omnipotents, surtout du roi de France. Ce dernier ne voulait pas se soumettre à une telle institution ou bien à une instance supérieure.

Que signifiait une telle fonction arbitrale dans la pratique politique? Le règne de l'empereur était-il vraiment une »hégémonie« ou ne restait-il qu'à appliquer la demande universelle pour se présenter en tant que médiateur (mediator/arbiter) dans les conflits internationaux - dans l'espoir que les parties du conflit acceptent son rang cérémoniel puisqu'il lui manquait tout moyen pour appliquer une sanction efficace?

II. Sigismond de Luxembourg comme roi romain germanique

\section{Est et Ouest}

Ce fut justement la situation dans laquelle s'est trouvé Sigismond de Luxembourg quand, après une double élection en 1410/1411, il devint roi romain germanique $^{18}$. Il était marié depuis 1375 avec la fille de Louis de Hongrie et de Pologne et, de ce fait, roi de Hongrie. Il a été confronté en Hongrie au plus grand défi de la politique internationale de ce temps: la pénétration militaire des Turcs/Osmans qui étaient devenus un danger latent à la frontière est de l'Europe et qui menaçaient en même temps Constantinople dans son existence même.

L'empereur byzantin essayait sans cesse de trouver de l'aide contre les Turcs et s'était pour cela personnellement présenté dans les cours de l'Europe de l'Ouest. L'une des images contemporaines les plus connues montre Sigismond en compagnie d'Eric du Danemark et de l'empereur Jean VIII. Paléologue lors d'une rencontre à Ofen. Placé au milieu des deux autres monarques, Sigismond se montrait comme empereur latin dans une position prédominante à côté de son homologue grec ${ }^{19}$. La concurrence de rang entre l'empereur ro-

18 Martin KINTZINGER, Sigmund (1410/1411-1437) mit Jobst von Mähren (1410/1411), dans: Bernd SCHNEIDMÓlLER, Stefan WeINFURTER (dir.), Die deutschen Herrscher des Mittelalters. Historische Portraits von Heinrich I. bis Maximilian I. (919-1519), Munich 2002, p. $462-485$.

${ }^{19}$ Le tableau et des explications sur les circonstances historiques: Jörg K. HoENSCH, Kaiser Sigismund. Herrscher an der Schwelle zur Neuzeit, 1368-1437, Munich 1996, p. 335sq. Sur la fonction politique des illustrations représentatives: Martin KINTZINGER, Imaginationen des Reiches, dans: Bernd SCHNEIDMÜlleR, Stefan WEINFURTER (dir.), Heilig - Römisch Deutsch. Das Heilige Römische Reich im mittelalterlichen Europa, Dresde 2006, p. 345371. 
main et byzantin avait une tradition de plus de six cents ans. Mais elle avait vu, au début du $\mathrm{XV}^{\mathrm{e}}$ siècle, son importance politique décroitre. La prédominance revendiquée de l'Empire occidental était encore davantage et avec beaucoup d'insistance visible. Le fait que Sigismond n'est couronné que neuf ans plus tard empereur ne nuit nullement au scénario. En fin de compte, il ne pouvait pas souligner son exigence en 1424 à Ofen par une supériorité de fait, mais seulement à cause de son rôle politique actuel: celui de médiateur entre l'Ouest et l'Est.

Quoi qu'il en soit, on ne pouvait pas attendre de la part des Latins une aide en faveur des Grecs contre les Turcs, la condition devait être une unité des Églises. Sigismond a mis en relief cette demande agréable aux Byzantins comme conditio sine qua non et il n'a pas par ailleurs proposé d'autre aide concrète. Il s'est prononcé pour le christianisme occidental en général, il a obtenu le soutien en Europe et ce programme monumental a presque fait oublier qu'il n'était fondamentalement pas en mesure de promettre ou d'obtenir plus ou de donner davantage de précisions. Il était assez réaliste pour savoir qu'il n'avait aucune chance de rallier les princes de l'Ouest à la défense de Constantinople. Dans le désir de s'imposer en tant qu'empereur universel, il pouvait cependant exiger l'union des Églises comme condition préalable pour l'aide demandée et comme tâche pour les conciles généraux de Constance et de Bâle comme l'avait fait l'Église romaine depuis longtemps. C'est en cela que réside son activité de médiateur entre l'Ouest et l'Est.

Cette revendication démonstrative à elle seule exerce son effet. Le sultan Murad II donne encore en 1424 son accord à une paix, afin de rassembler ses forces et pour empêcher l'union des chrétiens latins et grecs. C'était Sigismond qui a une grande part au succès de cette entreprise. Ces intentions politiques peuvent paraître - comme assez souvent dans les longues années de son règne mouvementé - spontanées et surprenantes. Mais elles ne l'étaient pas. S'il avait voulu agir selon les possibilités données par la politique de pouvoir appuyée sur la situation, il aurait dû rester tranquille. Mais il s'est décidé autrement et a suivi une conception politique très astucieuse: la présentation et la mise en scène représentative de l'autorité impériale d'un côté, et de l'autre la réalisation qui prend en considération la situation et qui est pragmatique. Le rôle en tant que médiateur - comme imperator et mediator - dont il se chargeait souvent et volontiers, était une des options d'activité possibles qui a découlé de cette conception. 


\section{Les souffrances de l'Est et la culture de l'Ouest}

Plus que d'autres, Sigismond avait acquis de l'expérience avec la médiation entre l'Ouest et l'Est. Longtemps avant son élection en tant que roi allemand et sans qu'une élévation en tant que roi ou empereur allemand soit visible, il avait déjà appliqué la même conception politique. Étant roi de Hongrie, il ne lui restait à la fin des années quatre-vingt-dix du XIV ${ }^{\mathrm{e}}$ siècle pas d'autre choix contre la menace pressante des Turcs à ses frontières orientales que d'appeler les princes de l'Ouest à l'aide. Étant dans une situation similaire à celle de l'empereur byzantin un quart de siècle plus tard, il choisit une autre stratégie. Il a envoyé ses attachés aux plus importantes des cours d'Europe occidentale, en particulier auprès de celles du royaume de France et du duché de Bourgogne. Ils ne devaient pas demander de l'aide pour la Hongrie qui n'était pas au centre de l'intérêt en tant qu'extrême poste oriental de l'Europe. De plus, la cour française avait renoncé à ses propres ambitions en Hongrie seulement quelques années avant en faveur des Luxembourg.

Le message des délégués hongrois était tout à fait différent: les Turcs menacent l'Occident chrétien, et après leurs victoires en Serbie, ils désireront conquérir et d'autres lieux de Hongrie, et de là se mettre à campagne acharnée contre l'Europe de l'Ouest. Une catastrophe menace l'Occident chrétien. Les princes de l'Ouest, doivent en tant que chrétiens et en tant que chevaliers arrêter pour cette raison l'avancée des Turcs ${ }^{20}$.

Même pour Sigismond le succès était surprenant. Les milites christiani d'Europe occidentale, surtout ceux venant de Bourgogne, se sont sentis obligés de faire leur devoir et ont proclamé un voiage en Hongrie, une aventure chevaleresque contre les Turcs ${ }^{21}$. Le fait que cette entreprise fut reconnue comme une croisade par les papes en pleine rivalité ne joua qu'un rôle secondaire. Encore au milieu du XV siècle la cour de Bourgogne s'est adonnée à un projet similaire, en bonne partie mais pas exclusivement dans un but de représentation ${ }^{22}$. C'était vraiment à cette époque le rassemblement d'une armée colossale qui s'est mise en route vers la Hongrie et qui sous la direction de Sigismond et du prince héritier de Bourgogne a rencontré en 1396 l'armée du sultan turc à Nicopolis. La fin est connue: n'ayant pas réussi à s'accorder sur une stratégie commune, ils sont tombés dans un piège tendu par les Turcs et ont péri. La majorité des nobles franco-bourguignons sont morts sur le

${ }^{20}$ KINTZINGER, Westbindungen (voir n. 8), p. 233-237.

${ }^{21}$ En détail: ID., Sigismond, roi de Hongrie et la croisade, dans: Jacques PAvrot, Martine Chauney-Bouillet (dir.), Actes du colloque international »Nicopolis« 1396-1996 [...], Dijon 1997 (Annales de Bourgogne 68 [1996]), p. 23-33.

${ }^{22}$ Heribert MÜLLER, Kreuzzugspläne und Kreuzzugspolitik des Herzogs Philipp des Guten von Burgund, Göttingen 1993 (Schriften der Historischen Kommission bei der Bayerischen Akademie der Wissenschaften, 51); Marie-Thérèse CARON, Denis CLAUZEL (dir.), Le banquet du Faisan. 1454: l'occident face au défit de l'empire ottoman, Arras 1997. 
champ de bataille ou ont été exécutés par la suite, le Bourguignon lui-même a été fait prisonnier.

Sigismond s'est engagé à rassembler des fonds dans toute l'Europe pour racheter le Bourguignon, lui-même indemne s'était enfui de Nicopolis et avait immédiatement repris son rôle de médiateur, maintenant en visant le paiement d'une rançon. Déjà pendant sa fuite de Nicopolis via Constantinople, il a continué - apparemment imperturbable à l'issue de la catastrophe - sa publicité pour une autre campagne contre les Turcs. Sa réputation en France était cependant et pour longtemps au plus bas: on le rendait coupable de l'issue de la bataille - certainement non sans raison, mais aussi pour cacher le rôle néfaste du prince bourguignon ${ }^{23}$.

\section{Imperium et regnum}

Ce ne fut qu'en 1433 que l'on couronna Sigismond empereur à Rome. Depuis son couronnement comme roi, vingt ans auparavant, il avait toujours propagé et essayé véritablement à plusieurs reprises d'entreprendre un voyage à Rome pour y être couronné empereur. Son projet a sans cesse échoué face aux nécessités de la politique européenne qui l'obligeaient à intervenir. Jusqu'à la fin du concile de Constance en 1417 et dans les années vingt son engagement dans la politique extérieure en Europe de l'Ouest a prédominé, après les affaires en Europe "centrale orientale« (Ostmitteleuropa) se sont imposées. Sigismond pouvait y prendre des décisions en tant que roi de Hongrie, plus tard aussi de Bohême. Dans ces cas, il a également cherché le soutien international, par exemple lors de la préparation de la croisade contre les Hussites à partir de 1431. À côté des possibilités d'exercice du pouvoir, il utilisait maintenant aussi les chances de négociations.

Vers 1430 , l'Italie devenait le centre de la politique extérieure de Sigismond, plus particulièrement les communes de l'Italie du nord. Elles le fêtaient comme empereur de la paix et essayaient d'utiliser la gloire de sa représentation en leur faveur, encore plus fortement lors du couronnement impérial en 1433. Mais ces manifestations n'ont pas dépassé le stade d'échange d'actes cérémonieux et de poésie panégyrique. En Italie du nord, plutôt qu'en Europe occidentale, l'imperator aurait pu trouver son propre champ d'activité. Les faits réels n'y permettaient cependant pas le développement d'une politique du pouvoir et il manquait les opportunités permettant de parvenir à des médiations heureuses.

${ }^{23}$ KINTZINGER, Westbindungen (voir n. 8), p. 246-248. 


\section{Politique occidentale et politique de paix}

À l'Ouest, une politique de médiation mise en scène extrêmement onéreuse devenait la caractéristique du temps de la régence de Sigismond. Comme d'habitude, il avait d'abord confirmé les contrats d'amitié et de soutien des temps de son père qui, chez Charles IV, exprimaient un lien prononcé avec la couronne de France et la maison des Valois. Sans héritier masculin, il lui aurait été impossible de poursuivre la politique dynastique de son père. Son unique fille, Élisabeth, née en 1409, devait servir aux intérêts des Luxembourg en Europe "centrale orientale" (Ostmitteleuropa) et elle a épousé Albrecht de Habsbourg, plus tard lui-même roi des Romains.

Il existait à cette époque deux instruments pour nouer et renforcer des relations extérieures: les mariages politiques éprouvés et - de plus en plus important - une politique d'alliance s'adaptant à la situation. Puisqu'il ne disposait pas de l'option du mariage, Sigismond s'est dès le début concentré sur les contrats d'alliance. De plus - paraît-il - il s'est empressé de ne pas apparaître dans les contrats comme parti intéressé ou comme parti de conflit, mais il voulait être le représentant d'intérêts supérieurs. Les liens de Charles VI avec la France (malgré son aventure anglaise en 1373), maintenus par Sigismond, ne pouvaient jamais être plus qu'une des variantes possibles.

Cette vue des choses ne peut en aucune façon s'expliquer par la préférence personnelle de Sigismond, mais par les événements du temps et par son pragmatisme politique. La menace des Turcs avait quelque peu perdu en urgence après Nicopolis parce que le sultan victorieux s'était retiré à la surprise de tous. Mais le danger demeurait malgré tout. Tant que l'Europe était déchirée par le schisme pontifical ainsi que par la guerre entre la France et l'Angleterre, et affaiblie par la multitude de conflits dans tous les royaumes européens occidentaux et également dans le royaume romain germanique, on n'était pas à même de contrecarrer les attaques et les provocations extérieures.

Le but prépondérant de la politique internationale était l'unité des princes de l'Ouest. De ce but ne dépendait pas seulement la possibilité d'une lutte contre les Turcs, mais aussi le succès urgent et nécessaire du concile qui se tenait à Constance depuis 1415 , aussi bien que le succès des négociations multiples de paix entre les princes d'Empire et entre les cours de France et d'Angleterre. On ne tendait pas à une paix éternelle, mais à l'interruption des conflits pour réunir les forces et pour mener à bien les tâches communes. On ne liait plus iustitita à pax, comme on l'avait fait depuis les temps anciens, mais à tranquillitas, la tranquillité interne sur laquelle on était tombé d'accord, l'armistice qui n'enrayait pas les conflits mais les suspendait permettant d'atteindre un but commun supérieur ${ }^{24}$. Ceci était le langage de Sigismond qui était directement

${ }^{24}$ Ibid., p. 348-359; Heinrich FINKE (dir.), Acta Concilii Constanciensis, t. 1: Akten zur Vorgeschichte des Konstanzer Konzils (1410-1414), Münster 1896 (réimpression Münster 
touché par les luttes contre les Turcs, comme bailli du concile et par les négociations de paix alors en cours. Il comprenait cependant sa tâche dans un contexte beaucoup plus ample. Peu de temps après l'ouverture du concile, il quittait pendant plus d'une année le lieu de la réunion pour se mettre en route à travers l'Europe occidentale. Ce voyage visait à apaiser les rois de France et d'Angleterre. Il avait projeté un modèle pour cet objectif: une alliance fraternelle des rois de France et d'Angleterre et de l'Empire romain germanique, un foedus fraternitatis, qui devait réunir les trois monarques comme dirigeants $\mathrm{du}$ christianisme.

\section{Médiation et arbitrage}

Sigismond aurait-il pu mettre fin à la guerre entre la France et l'Angleterre qui se déroulait depuis déjà trois générations et qui durera encore un demi siècle et qui sera plus tard nommée la guerre de Cent Ans? La conclusion d'une paix définitive était impossible à atteindre et ce n'était pas le but de la politique de Sigismond. Il voulait tenter d'être un des maillons dans les négociations interminables de paix ou mieux dans les négociations sur l'armistice entre les deux partis. Il espérait pouvoir atteindre comme médiateur indépendant ayant un intérêt plus élevé (l'unité des chrétiens capable de prendre des décisions solides, l'unité de l'Église et de l'Europe) une interruption des luttes qui durerait au moins quelques années - assez longtemps pour terminer le concile et obtenir l'élection du Pape, peut-être aussi préparer une nouvelle guerre contre les Turcs.

À l'aide de mesures fiscales et économiques forcées ou par la menace de sanctions, il s'est servi de la politique du pouvoir à l'intérieur de l'Empire romain germanique pour ordonner une paix. Il voulait contraindre les princes belligérants de »son« royaume à conserver la paix afin que le royaume et le christianisme puissent agir en commun contre leurs adversaires. Mais même cette politique n'était pas toujours couronnée de succès, car elle s'appuyait finalement sur une fiction: la présomption que le roi et le futur empereur en tant que chef du royaume sera en mesure d'exiger des actions efficaces ou la

1976), Die Verhandlungen Sigismunds mit Karl VI. von Frankreich, der Pariser Universität und Heinrich V. von England. Winter 1413 und Herbst 1414, p. 358-391; $n^{\circ} 104$ (1414), p. 377, 1. 15-20: [...] dominus rex Romanorum causa et amore reipublice tam ecclesiastice quam secularis ac mutae confederationis et amicicie peramplius solidande maxime flagrat affectu, ut, ex quo inter reges et principes catolicos orbis terre Romanorum, Francorum et Anglie reges reputati sunt et existunt tamquam tria precipua capita in christianitate [...]. Autres preuves: Dieterich KERLER (dir.), Deutsche Reichstagsakten unter Kaiser Sigmund, erste Abteilung, 1410-1420, D. Das Bündnis mit England gegen Frankreich, Munich 1878 (Deutsche Reichstagsakten, 7), p. 332-345. 
renonciation - seulement sur la base de l'autorité de son rang et (mis à part sa position prédominante féodale) sans moyen de pouvoir ou de contrainte.

En politique extérieure cette question était depuis longtemps réglée. Dans les années vingt, il a essayé de faire adopter des droits féodaux par une politique du pouvoir contre les ducs de Bourgogne et de Brabant. Il les a cités devant son tribunal pour cause d'infraction à la constitution royale et à l'ordre féodal. C'était sûrement sans le vouloir qu'il a contribué au fait que l'exercice du pouvoir des princes de l'Europe de l'Ouest contre les vieilles règles de la maison régnante et les exigences de supériorité de l'Empire romain soit dévoilé à la vue de tout le monde. Il est bien évident que les interpellés n'ont pas comparus devant son tribunal.

On ne doit y voir qu'une accentuation rituelle des droits perdus. Aux débuts de son gouvernement, il avait épuisé un plus ample répertoire de possibilités, surtout lors de l'épisode de son engagement en France entre 1412 et 1417. Sigismond n'avait aucune possibilité d'influencer d'une manière quelconque les rois de France ou d'Angleterre ou bien encore les grands princes de son Empire par son propre pouvoir. Il ne pouvait faire jouer que son autorité impériale et son efficacité dépendait largement de l'acceptation de celle-ci par ses interlocuteurs. Des défilés solennels et des réceptions, des banquets de gala ou des chasses et autres réjouissances plaisaient aux cours de l'Occident. La splendeur de la dignité impériale que Sigismond ne possédait pas encore en réalité et qu'il disait ne pas vouloir retombait quand même sur les hôtes. Pendant ses voyages à travers l'Europe en 1415/1416 Sigismond était souvent un invité bienvenu et seulement quelques communes refusèrent l'entrée de l'invité parce qu'elles craignaient les frais énormes d'entretien, surtout face aux faibles perspectives de gain politique.

Dans quelle mesure il pouvait faire valoir ses intentions d'action, ceci est un autre chapitre. Son désir d'agir en tant que mediator est à peine pris au sérieux à Paris et Londres - mais cela n'en resta pas seulement là, pour la »belle« gloire. Des conversations sérieuses ont eu lieu et se sont soldés par des succès. À la suite d'un entretien personnel avec Sigismond, Ferdinand d'Aragón 'à été le premier à être prêt à abandonner de l'obédience avignonnaise et ainsi permettre la nouvelle élection d'un pape. En fin de compte les rois de France et d'Angleterre ont suivi son exemple, mais seulement après une assez longue préparation.

Le succès rapide avait-il amené Sigismond à devenir insouciant ou voulait-il tout simplement exiger le maximum afin d'obtenir un minimum comme c'est le cas habituel en diplomatie? En 1415, il a expliqué au roi d'Angleterre et à la réunion du concile qu'il désirait obtenir la paix entre la France et l'Angleterre grâce à sa médiation et celle d'autres personnes appropriées, qu'il désirait même obtenir la paix de tous les rois et princes du christianisme (mediantibus 
nobis et aliis personis ydoneis [...] ad pacem omnium regnorum et principum christianitatis). Sigismond se considérait comme tantus bonus mediator ${ }^{25}$.

Durant l'été 1416, il a fait part de sa déception en ce qui concerne la cour française qui, sous sa direction - malgré des conversations intenses durant des mois accompagnés de promesses magnifiques - ne voulait pas faire d'offre de paix à l'Angleterre. S'était-il vraiment attendu à autre chose? On parlait même volontiers avec lui d'un grand plan de paix, mais sa réalisation n'était jamais sérieusement prise en considération - aussi bien du côté français que du côté anglais.

Ce n'est qu'à première vue que cette réaction semble contradictoire. Elle met l'accent sur le fait que la politique de médiation de Sigismond a été prise au sérieux. On acceptait le futur empereur en tant que médiateur (mediator), mais on voulait et on devait éviter qu'il devienne un arbitre (arbiter). On pouvait recevoir le mediator dans le cadre mineur d'une mise en scène représentative et on pouvait également négocier sérieusement avec lui car il était (selon la définition et la chose) un partenaire neutre entre les partis auquel on concédait une dignité cérémonielle qui était différente de son rang. Un arbiter par contre pouvait exiger de trancher réellement entre les partis, de rendre un arbitrage et d'assurer ainsi un rang supérieur aux partis. Une mediatio ne touchait en aucun cas à l'indépendance des partis, un arbitrium fait déjà percevoir étymologiquement qu'il pouvait comprendre un acte décisionnel voulu par l'arbitre ${ }^{26}$. Dans les royaumes de l'Europe occidentale, les rois se considéraient comme imperatores in regnorum suorum et c'est pour cette bonne raison qu'ils ne pouvaient pas accepter un tel arbitrium, de même que tout autre politique hégémonique ou toute exigence de supériorité.

On peut certainement supposer que Sigismond était conscient de la différence entre les deux formes de procédure et qu'il aspirait non seulement au rôle de médiateur, mais il voulait agir en tant qu'arbitre. Là où il était, grâce à l'exercice du pouvoir, à même de se charger du rôle d'arbitre, comme par exemple sur ses propres terres, il utilisait volontiers ses possibilités et cela avec insistance. Mais il ne se contentait pas d'actes hégémoniques, mais il présentait, dans la mesure du possible, les raisons de son activité par un travail diplomatique préalable astucieux. Son approche - analysée par Alain Demurger - d'un parti de paix qui a travaillé pendant peu de temps à la cour française entre 1412 et 1414 en est une preuve impressionnante. Même l'accolade qui fit fureur au parlement de Paris en 1416 s'insère dans ce contexte. Il devait avoir des connaissances considérables sur les liens personnels à l'intérieur de la cour

${ }^{25}$ KINTZINGER, Westbindungen (voir n. 8), p. 98. Instructives les indications, dans: FINKE (dir.), Acta Concilii Constantiensis (voir n. 24). Preuve exemplaire: p. 362, 1. 12 (oratores sive mediatores, 1414).

${ }^{26}$ Voir Helmut G. WALTHER, Imperiales Königtum, Konziliarismus und Volkssouveränität. Studien zu den Grenzen des mittelalterlichen Souveränitätsgedankens, Munich 1976, p. 184. 
française. Vivement critiquées par beaucoup de gens, ces négociations se déroulaient avec l'autorisation tacite, pour ne pas dire avec l'accord du roi. Elles ne représentaient pas une usurpation des droits royaux en France par le futur empereur - pas plus qu'une politique d'hégémonie - mais montraient d'une manière symbolique l'appui efficace au parti d'un roi affaibli.

Le fait que la situation à la cour d'un Charles VI, malade, et les intrigues entre les partis (avec une fin souvent mortelle) était trop complexe pour permettre une orientation à long terme qui soit efficace, a fait finalement échouer l'approche bien préparée de Sigismond depuis 1414 et jusqu'en 1416. Son rapprochement apparemment surprenant en direction de l'Angleterre, qui finit en été 1416 avec la conclusion du contrat de Canterbury, a suivi les mêmes règles: préparation diplomatique minutieuse et intention déclarée d'agir en tant que mediator. Soi-disant en réaction au refus de la France d'accepter ses intentions de médiation, le contrat a été conclu avec Henri d'Angleterre. Il n'a jamais été considéré comme un pacte de soutien contre la France et ne pouvait à aucun moment compter sur l'appui des princes d'Empire. Il faut le lire comme un signe: quand sa mission n'est pas acceptée, le mediator est libre de se mettre du côté d'un parti. En fin de compte on voulait ouvrir par la provocation les portes vers de nouvelles conversations de médiation. Ce plan échoua et peut être considéré comme conséquence de la tactique de Sigismond. Avec la conclusion du contrat de Canterbury il a abandonné son rôle de mediator et il a agi de manière »arbitraire« et prenant parti comme arbiter.

Cette différenciation, comme d'ailleurs la politique de médiation comme option préférée de la politique extérieure de Sigismond en Europe de l'Ouest au début de son règne, montre les traits d'une redécouverte contemporaine de la manière de penser en droit des gens. Sigismond lui-même n'a pas utilisé la notion et la compréhension du droit des gens dans ses documents et ses publications politiques - mais il en a fait ressortir les conceptions respectives.

Il est impossible de prouver que Sigismond ait eu des connaissances sur la nouvelle forme de pensée sur le droit des gens à son époque. Mais on peut être sûr qu'il a dû connaître - en tant que président du concile de Constance - les différences entre l'Ordre allemand et la Pologne, avant tout les explications de maître Paulus Vladimiri de Krakau sur le traitement des peuples païens en 1415. Le texte de Vladimiri - présenté et publié au cours du concile - appartient aujourd'hui aux témoignages importants concernant le changement de la manière de penser en droit des gens autour de 1400 .

Les commentaires légaux sur le droit des gens comme ils ont été rédigés dans les universités italiennes à la fin du XIV ${ }^{e}$ siècle et qui traitent en particulier de la médiation à l'occasion de conflits internationaux et du rôle de l'empereur, ne lui sont certainement pas inconnus. Aucun autre empereur de la fin du Moyen Âge, pas même Charles IV, n'a appelé autant de vassaux italiens à sa cour que Sigismond. La proportion de gradués universitaires était extraor- 
dinairement élevée. Parmi ses familiares italiens, il y a deux hommes de Florence, Padoue et Pérouse (jadis lieux des activités de Baldus de Ubaldis), qui déjà entre 1411 et 1413 sont venus à la cour de Sigismond, l'un docteur en droit romain ${ }^{27}$. Mais on ne peut cependant pas en conclure que ces familiares aient apporté des connaissances des écritures de Baldus à la cour de Luxembourg; d'autre part, il ne faut pas d'attestation d'origine des villes universitaires nommées, pour prendre en considération un effet des textes de Baldus à la cour des Luxembourg. Chaque légiste et diplomate expérimenté et d'autant plus chaque Italien érudit des familiares de Sigismond peut avoir publié les textes des grands légistes comme Bartolus de Sassoferato et Baldus de Ubaldis qui sont en général commentés dans le monde érudit du temps ${ }^{28}$. Il est très probable que Sigismond - particulièrement érudit et toujours ouvert aux nouveautés - se soit approprié de tels documents utiles à sa politique pragmatique.

Il s'ensuit au moins que Sigismond, dans sa politique occidentale, se soit servi d'un rôle politique et ait formé son intention d'agir comme médiateur international avec l'autorité impériale comme il est conçu dans les documents juridiques sur le droit des gens. Sa politique pragmatique entre pouvoir et droit devenait donc un champ d'exercice pour l'application des conceptions du droit interne des gens. Celles-ci ne pouvaient être couronnées de succès que dans la mesure où elles offraient une médiation et ne pénétraient pas dans le domaine de l'arbitrage international. Ceci se voit dans la politique occidentale de Sigismond. Encore en 1462, le plan de paix ambitieux du roi de Bohême, Georges Podiebrad (Poděbrad), échouera à cause de cet argument dans la pratique politique, malgré sa modernité théorique surprenante.

${ }^{27}$ Laurentius Jacobus de Gentelottis et Simeon, le dernier Doctor legum: KINTZINGER, Westbindungen (voir n. 8), annexe, p. 434, 459, et Gisela BEINHOFF, Die Italiener am Hof Kaiser Sigismunds (1410-1437), Francfort/M. 1995 (Europäische Hochschulschriften, Reihe 3, 620 ), $\mathrm{n}^{\circ}$ 295, 497; aussi Johannes Bello (Padua): KINTZINGER, Westbindungen (voir n. 8), p. 421, et BEINHOFF, Italiener, $n^{\circ}$ 67; Philipp del Bene (Florence): KINTZINGER, Westbindungen (voir n. 8), p. 421, et BEINHOFF, Italiener, $n^{\circ} 68$; Johannes Francisci de Herricis (Florence): KINTZINGER, Westbindungen (voir n. 8), p. 437, et BEINHOFF, Italiener, $n^{\circ} 317$; Mandolus Franchi (Padua): KINTZINGER, Westbindungen (voir n. 8), p. 441.

${ }^{28}$ Sur la théorie politique de Bartolus: WALTHER, Königtum (voir n. 26), p. 176-186. 
